ARTICLE

Received 24 Jun 2015 | Accepted 10 Dec 2015 | Published 3 Feb 2016

DOI: $10.1038 /$ ncomms10454

OPEN

\title{
Absence of a spin-signature from a single Ho adatom as probed by spin-sensitive tunneling
}

\author{
M. Steinbrecher ${ }^{1}$, A. Sonntag ${ }^{1}$, M. dos Santos Dias ${ }^{2}$, M. Bouhassoune ${ }^{2}$, S. Lounis ${ }^{2}$, J. Wiebe , \\ R. Wiesendanger ${ }^{1} \&$ A.A. Khajetoorians ${ }^{1,3}$
}

Whether rare-earth materials can be used as single-atom magnetic memory is an ongoing debate in recent literature. Here we show, by inelastic and spin-resolved scanning tunnellingbased methods, that we observe a strong magnetic signal and excitation from Fe atoms adsorbed on Pt(111), but see no signatures of magnetic excitation or spin-based telegraph noise for Ho atoms. Moreover, we observe that the indirect exchange field produced by a single $\mathrm{Ho}$ atom is negligible, as sensed by nearby Fe atoms. We demonstrate, using ab initio methods, that this stems from a comparatively weak coupling of the Ho $4 f$ electrons with both tunnelling electrons and substrate-derived itinerant electrons, making both magnetic coupling and detection very difficult when compared to $3 d$ elements. We discuss these results in the context of ongoing disputes and clarify important controversies.

\footnotetext{
${ }^{1}$ Department of Physics, Hamburg University, 20355 Hamburg, Germany. ${ }^{2}$ Peter Grünberg Institut and Institute for Advanced Simulation, Forschungszentrum Jülich \& JARA, 52425 Jülich, Germany. ${ }^{3}$ Institute for Molecules and Materials (IMM), Radboud University, 6525 AJ Nijmegen, The Netherlands. Correspondence and requests for materials should be addressed to S.L. (email: s.lounis@fz-juelich.de) or to J.W. (email: jwiebe@physnet.uni-hamburg.de) or to A.A.K. (email: a.khajetoorians@science.ru.nl).
} 
sing the magnetic moment of a single atom adsorbed on non-magnetic surfaces to store and process information is one of the major goals in the field of nano-spintronics ${ }^{1}$. A key ingredient towards realizing single-atom magnets is the large magnetic anisotropy energy ${ }^{2}$, which defines an energy barrier between various orientations of the magnetic moment and has been found to be extraordinarily large for single $3 d$ atoms, like Co, adsorbed directly on metallic surfaces ${ }^{3}$. However, strong hybridization of the $3 d$ states makes the magnetic moment susceptible to substrate-driven interactions, like spin-flip scattering by conduction electrons ${ }^{4}$, diminishing the stability of the magnetic moment against fluctuations. One route towards increasing the magnetic stability of single-atom magnets is to use strong exchange coupling between a small number of magnetic atoms, thereby increasing the effective energy needed to reverse the magnetization of the atomic ensemble. Towards this end, it was demonstrated that a small number of strongly coupled $\mathrm{Fe}$ atoms in an array ${ }^{5}$ or a chain ${ }^{6,7}$, either ferro- or antiferromagnetically coupled, can be controllably stabilized into a given state from seconds to hours.

It was also proposed that, by combining a particular choice of symmetry, magnetic moment and strong uniaxial anisotropy, the spin can be protected from quantum tunnelling of the magnetization and substrate-driven relaxation ${ }^{8-10}$. Experimentally, based on this concept, Miyamachi et al. ${ }^{8}$ claim to stabilize a single Ho atom on $\mathrm{Pt}(111)$. This realization is motivated by a key property of bulk $4 f$ magnetism; the spin resides in the $4 f$ orbitals, which are strongly localized to the nucleus and only couple to the itinerant electrons via intra-atomic exchange paths through the $5 d$ - and $6 s$-orbitals ${ }^{11}$ and therefore the $4 f$ orbitals only negligibly contribute to the tunnelling current ${ }^{12}$. This property of $4 f$ elements hypothetically allows for a weaker degree of hybridization as compared with the $3 d$ counterparts, when adsorbed onto metallic surfaces. However, there has been recent controversy about this claim, stemming from disagreement about the magnetic properties of $\mathrm{Ho} / \mathrm{Pt}(111)$. X-ray magnetic circular dichroism (XMCD) measurements ${ }^{13}$ reveal no evidence of magnetic stability and a different ground state configuration for $\mathrm{Ho} / \mathrm{Pt}(111)$ compared with ref. 8, which violates the theoretical criterion proposed by Miyamachi et al. ${ }^{8}$.

To clarify this controversy and understand if tunnelling-based spectroscopy can reveal the magnetism of $4 f$ elements, we revisit Ho atoms on $\mathrm{Pt}(111)$ with spin-resolved tunnelling spectroscopy at $T=0.3 \mathrm{~K}$. In stark contrast to what is reported by Miyamachi et $a{ }^{8}{ }^{8}$, we see no evidence of spin-excitations or spin-polarized telegraph noise on these atoms. The findings were corroborated by concurrently performed experiments with $\mathrm{Fe}$ atoms on the same surface, where the magnetic properties of this system are well-known ${ }^{14}$ and confirm our spin-sensitivity. Furthermore, by monitoring the changes in spin-excitations of a single Fe atom, we find that the indirect exchange fields produced by a nearby Ho atom cannot be detected. These findings, as illustrated by $a b$ initio methods, stem from a weak interaction of the $4 f$ orbitals with the surrounding electronic environment, which thereby makes the Ho magnetic moment difficult to access by both tunnelling methods and indirect exchange pathways.

\section{Results}

Inelastic tunnelling spectroscopy of single $\mathrm{Ho}$ and $\mathrm{Fe}$ atoms. In constant-current scanning tunnelling microscopy (STM) images, $\mathrm{Ho}$ and $\mathrm{Fe}$ atoms can easily be distinguished by their different apparent heights, which are $\approx 180 \mathrm{pm}$ for Ho and $\approx 120 \mathrm{pm}$ for Fe atoms, respectively (Fig. 1a,b). We observe that the apparent height of the Ho atom depends strongly on the bias voltage $V_{\mathrm{s}}$ applied to the sample with a maximum of $\approx 205 \mathrm{pm}$ at $V_{\mathrm{s}}=1.5 \mathrm{~V}$
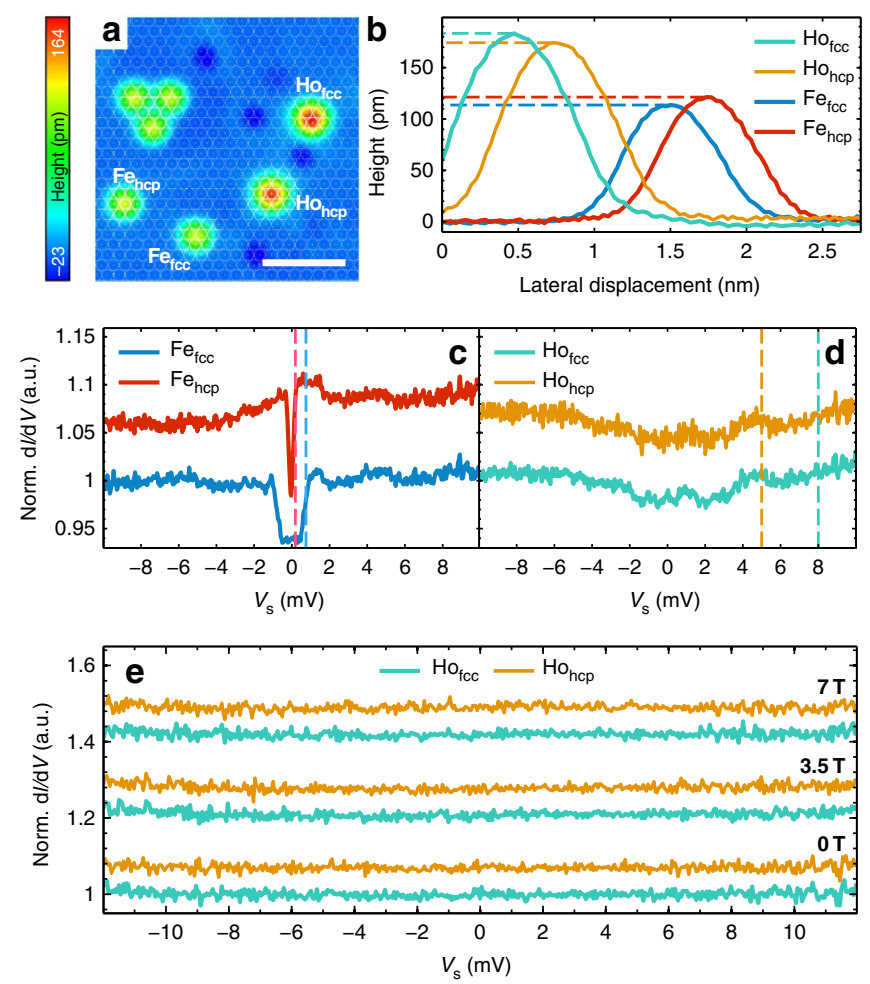

Figure 1 | Topography and inelastic spectroscopy of a single Fe and Ho

atom. (a) Constant-current image of an artificial arrangement of $\mathrm{Fe}$ and $\mathrm{Ho}$ atoms on fcc and hcp lattice sites $\left(V_{s}=10 \mathrm{mV}, I_{\text {stab }}=0.5 \mathrm{nA}\right)$. The overlaying white lattice has the lattice constant and orientation of the $\mathrm{Pt}$ (111) substrate but is centred on the $\mathrm{Fe}_{\mathrm{hcp}}$ species and reveals the different adsorption sites of the atoms. The white scale bar has a length of $2 \mathrm{~nm}$ and the colour scale on the left side indicates the measured apparent height in a range of -23 to $164 \mathrm{pm}$. (b) Line profiles of the atoms in a taken from a topography recorded at $V_{s}=-50 \mathrm{mV}$ and $I_{\text {stab }}=100 \mathrm{pA}$. The dashed lines indicate the dedicated maximum heights. (c,d) $d / / d V$ spectra measured on the isolated $\mathrm{Fe}$ and $\mathrm{Ho}$ atoms of a using the same microtip. The dashed lines indicate previously reported spin-excitation energies ${ }^{5,8}$ $\left(I_{\text {stab }}=5 \mathrm{nA}, V_{\text {stab }}=10 \mathrm{mV}, V_{\text {mod }}=40 \mu \mathrm{V}, f_{\text {mod }}=4.142 \mathrm{kHz}, \tau=10 \mathrm{~ms}\right.$ and $V_{\text {sens }}=10 \mathrm{mV}$ ). (e) Magnetic field-dependent $\mathrm{d} / \mathrm{d} V$ spectra measured on an isolated $\mathrm{Ho}$ atom at the indicated $B_{z}$ values and settings as in $\mathbf{d}$ except for $I_{\text {stab }}=6 \mathrm{nA}, V_{\text {stab }}=12 \mathrm{mV}$. Spectra in $\mathbf{d}, \mathbf{e}$ have been normalized by subtraction of a substrate spectrum taken with the same microtip and are artificially offset for clarity.

(see Supplementary Note 1 and Supplementary Figure 2), in excellent agreement with the results of Donati et al. ${ }^{13}$. These values significantly deviate from what was published by Miyamachi et al. ${ }^{8}$, where apparent heights above $220 \mathrm{pm}$ $\left(V_{s}=1 \mathrm{~V}\right)$ have been reported. We find small differences in the apparent height for fcc and hcp atoms. Interestingly, the $\mathrm{Fe}_{\mathrm{hcp}}$ atoms appear higher than $\mathrm{Fe}_{\mathrm{fcc}}$, whereas $\mathrm{Ho}$ atoms show the opposite behaviour (Fig. 1b). With a given tip, inelastic scanning tunnelling spectroscopy (ISTS) on the Fe atoms reveals the previously reported ${ }^{14}$ adsorption-site-dependent spectroscopic signature of a spin-excitation with a different energy for $\mathrm{Fe}_{\mathrm{fcc}}$ and $\mathrm{Fe}_{\mathrm{hcp}}$ sites. They are located at $0.75 \mathrm{meV}$ and $0.19 \mathrm{meV}$ (dashed lines in Fig. 1c), with step heights of $8 \%$ and $12 \%$ of the signal at zero bias, respectively. Knowing the adsorption site, tipinduced atom manipulation has been used to assemble artificial arrangements of $\mathrm{Ho}_{\mathrm{fcc}}, \mathrm{Ho}_{\mathrm{hcp}}, \mathrm{Fe}_{\mathrm{fcc}}$ and $\mathrm{Fe}_{\mathrm{hcp}}$ on clean areas of the $\mathrm{Pt}(111)$ substrate, which we used for subsequent investigations of their magnetic properties (Fig. 1). Typical manipulation 
parameters are $V_{\mathrm{s}}=2 \mathrm{mV}$ at a stabilization current of $I_{\text {stab }}=50 \mathrm{nA}$ for Fe, while Ho already moves at $30 \mathrm{nA}$.

We first performed magnetic field (B)-dependent ISTS and spin-resolved measurements on isolated Ho atoms which were far from other atoms. Using the same microtip as for ISTS on Fe (Fig. 1c) the differential tunnelling conductance $(\mathrm{d} / / \mathrm{d} V)$ signal on $\mathrm{Ho}_{\mathrm{fcc}}$ and $\mathrm{Ho}_{\mathrm{hcp}}$ is featureless and shows no reproducible inelastic signal distinguishable from the substrate spectrum (Fig. 1d). In particular, there is no indication for a spinexcitation at the previously reported energies of $5 \mathrm{meV}$ and $8 \mathrm{meV}$ for $\mathrm{Ho}_{\mathrm{fcc}}$ and $\mathrm{Ho}_{\mathrm{hcp}}$, respectively ${ }^{8}$ (see dashed lines in Fig. 1d). Moreover, the spectra in an energy window of $\pm 12 \mathrm{meV}$ do not change in a magnetic field up to $7 \mathrm{~T}$ (Fig. 1e). This rules out that spin-excitations emerge in a magnetic field at low energy due to negligible magnetic anisotropy ${ }^{15}$. We performed the same experiment with several tens of different microtips and atoms with stabilization currents up to $50 \mathrm{nA}$ and modulation voltages reaching $3 \mathrm{mV}$ using a second lock-in amplifier to measure the second derivative signal in parallel to $(\mathrm{d} I / \mathrm{d} V$ ) (see Supplementary Note 2), to directly compare with the method used by Miyamachi et $a l^{8}$. Several spectra with different parameters have been taken and are shown exemplarily in Supplementary Figures 3-5 in Supplementary Note 2. The noise level in these measurements was $\pm 0.5 \mu \mathrm{AV}^{-2}$ (see Supplementary Note 2). Nevertheless, we did not see any inelastic signatures unique to the Ho atom, regardless of the various parameters. It is important to note that tip-related features which appear as variations in the substrate spectra could not be fully removed from the spectra measured on the atoms by subtracting a substrate spectrum measured with the same tip. For all microtips we used, the minimum signal variation due to this effect was $\pm 2 \mu \mathrm{AV}^{-2}$ (see Supplementary Note 2). We remark, however, that the intensity of the inelastic signal was not specified by Miyamachi et al. ${ }^{8}$.

Spin-resolved measurements of $\mathrm{Ho}$ and $\mathrm{Fe}_{3}$. As XMCD measurements clearly indicate the presence of a magnetic moment of $\mathrm{Ho}$ on $\mathrm{Pt}(111)^{13}$, the lack of ISTS signal questions if the tunnelling electrons sufficiently exchange couple with the $4 f$ derived magnetic moment of the Ho atom ${ }^{16,17}$. Another approach to probe this is to employ spin-resolved tunnelling, which is sensitive to the spin-polarization in the vacuum ${ }^{18}$. To perform such measurements, we created a magnetic tip by intentionally picking up $\mathrm{Fe}$ atoms from the substrate to the tip, until spinsensitivity was achieved. The spin-sensitivity to the out-of-plane component of the magnetization was quantified by recording the magnetic telegraph noise of the tip height $\Delta Z$ on an artificially constructed array of three Fe atoms ( $\mathrm{Fe}_{3}$-array, Fig. 2a,b). The magnetization of this array switches between two degenerate ground states with opposite out-of-plane magnetization components (red and blue in Fig. 2b) similar to $\mathrm{Fe}_{5}$ on $\mathrm{Cu}(111)^{5}$, as proven by an increase in the asymmetry of the residence times in the two states with $B$. The observed telegraph noise has a strength of $\Delta Z \approx 15 \mathrm{pm}$ on a noise level of $0.3 \mathrm{pm}$ (r.m.s., time averaging $20 \mathrm{~ms}$ ).

Using the $\mathrm{Fe}_{3}$ as a calibration, we employ tips which showed the above-mentioned contrast to probe individual Ho atoms (Fig. 2b,c). Time traces with calibrated tips and various combinations of the parameters $I_{\text {stab }}(0.5$ to $50 \mathrm{nA}), V_{\mathrm{s}}$ (3 to $10 \mathrm{mV}$ ), $B(-0.2$ to $0.2 \mathrm{~T}$ ) and recording times $t$ (200 to $1,600 \mathrm{~s}$ ), which include the choices of parameters of Miyamachi et al. ${ }^{8}$, have been recorded on 13 different Ho atoms (see Supplementary Table 1 in Supplementary Note 3 for the list of parameter combinations). The 39 time traces (Fig. 2d) do not show any indication for the magnetic telegraph noise that has been reported in ref. 8, within a noise level specified above. Recent
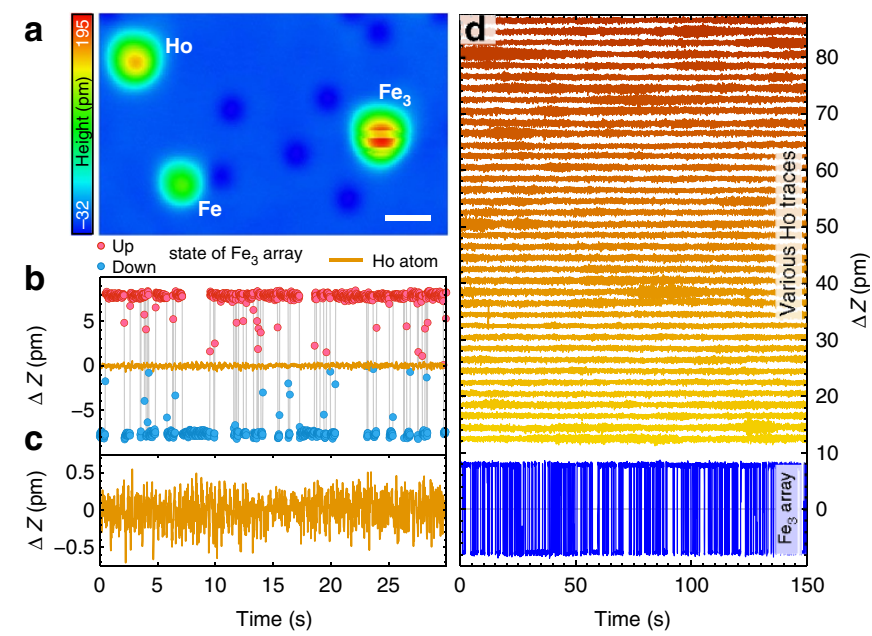

Figure 2 | Spin-polarized measurements on single Ho atoms and an $\mathbf{F e}_{\mathbf{3}}$-array. (a) Constant-current image of an $\mathrm{Fe}_{3}$-array, an $\mathrm{Fe}$ and a $\mathrm{Ho}$ atom $\left(V_{\mathrm{s}}=-5 \mathrm{mV}\right.$ and $\left.I_{\text {stab }}=0.5 \mathrm{nA}\right)$ recorded with a spin-polarized tip. The height of the $\mathrm{Fe}_{3}$-array ( $\approx 200 \mathrm{pm}$ ) changes randomly while recording the image due to switching of its magnetization. The white scale bar has a width of $1 \mathrm{~nm}$ and the colour scale on the left side indicates the measured apparent height in a range of -32 to $195 \mathrm{pm}$. (b) Time trace of the magnetic telegraph noise of the $\mathrm{Fe}_{3}$-array (colours red and blue represent the two magnetization states up and down) and of a Ho atom recorded with the same magnetic tip (orange line) $\left(V_{\mathrm{s}}=5 \mathrm{mV}, I_{\text {stab }}=1 \mathrm{nA}\right.$ and $B=0.2 \mathrm{~T}$ ). The magnetic tip is sensitive to the out-of-plane magnetization component. (c) Time trace of the Ho atom from $\mathbf{b}$ shown in a more narrow $\Delta Z$ range. (d) Thirty-nine time traces on 13 different Ho atoms (colour gradient from yellow to orange) recorded with spin-polarized tips and various parameters $I_{\text {stab }}(0.5$ to $50 \mathrm{nA}), V_{\mathrm{s}}(3$ to $10 \mathrm{mV}), B(-0.2$ to $0.2 \mathrm{~T})$ and recording times $t$ (200 to 1,600 s) (see Supplementary Note 3 for a list of parameter combinations). The telegraph noise of the $\mathrm{Fe}_{3}$-cluster (blue) from $\mathbf{b}$ serves as a reference.

measurements using XMCD reported a mixture of $J_{z}= \pm 6$ with an almost quenched expectation value along the surface normal as the magnetic ground state of Ho on $\operatorname{Pt}(111)^{13}$. While this would explain the observed absence of telegraph noise in our magnetic measurements in contrast to Miyamachi et $a .^{8}$, the corresponding spin-Hamiltonian still enables spinexcitations at a few $\mathrm{meV}$ energy, which we do not observe. Therefore, the lack of inelastic and spin-polarized signal from Ho questions if tunnelling-based methods are sensitive enough to interrogate the magnetism of $4 f$ single-atom moments. We discuss the latter point in more detail later, by comparing the experimental observations with the calculated electronic structure.

Study of the magnetic interaction between Ho and $\mathrm{Fe}$ atoms. It is well-known that magnetic order of bulk $4 f$ materials is mediated by indirect exchange interactions via exchange of the $4 f$ electrons with $5 d$ and $6 s$ itinerant electrons ${ }^{11}$. At the single-atom limit, it is interesting to ask if the exchange fields produced by single Ho atoms on surfaces behave similar to the bulk. Therefore, we use an $\mathrm{Fe}$ atom in close proximity to $\mathrm{Ho}$ and monitor the changes of the magnetic excitation of $\mathrm{Fe}$, to probe the indirect exchange field produced by Ho. We have shown in an earlier publication that the $\operatorname{Pt}(111)$ substrate mediates an RKKY interaction between two Co atoms adsorbed on the surface with a strength on the order of hundreds of $\mu \mathrm{eV}$ for pair separations in the range of $10 \AA$ (ref. 19). Using atom manipulation, we have 
a
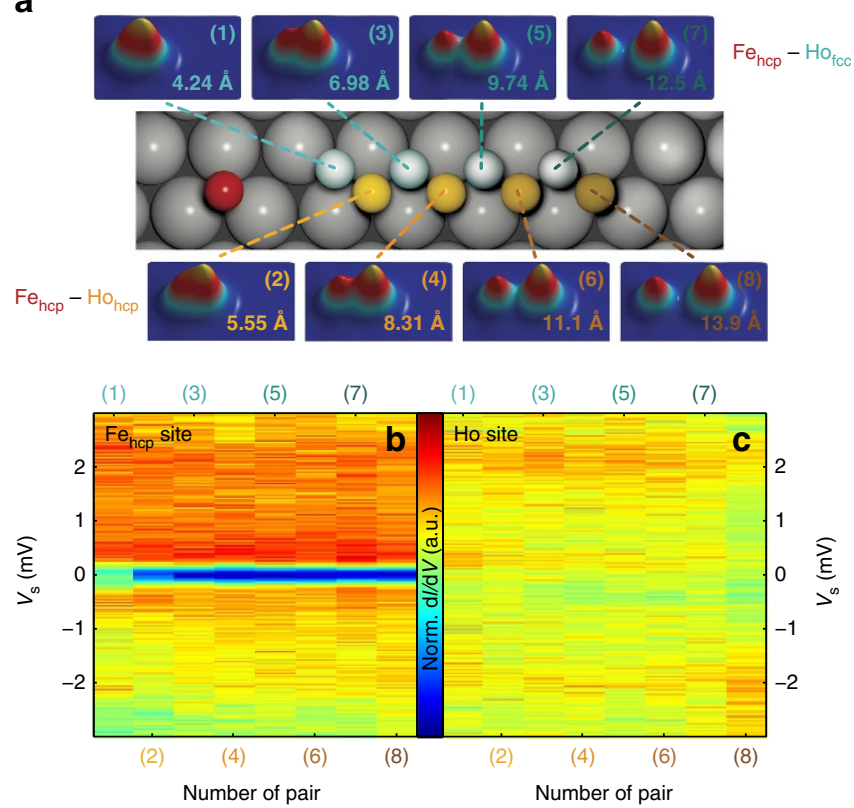

Figure 3 | Distance dependency of the magnetic interactions in an Fe-Ho pair. (a) Constant-current images $[(1-8)]$ of different $\mathrm{Fe}_{\mathrm{hcp}}-\mathrm{Ho}$ atom pairs with indicated distances and related ball-models in between. (b) Spectra on the $\mathrm{Fe}_{\mathrm{hcp}}$ atom and (c) the Ho atom for each pair with the dl/dV signal represented by the given colour scale. The spectra have been normalized to a substrate spectrum, divided by their mean value and the colour scaling for Fe and $\mathrm{Ho}$ is the same $\left(I_{\text {stab }}=3 \mathrm{nA}, V_{\text {stab }}=10 \mathrm{mV}\right.$ and $\left.V_{\text {mod }}=40 \mu \mathrm{V}\right)$.

built several pairs containing one fixed $\mathrm{Fe}_{\mathrm{hcp}}$ atom and a Ho atom that was moved to different lattice sites (both fcc and hcp), resulting in various distances between the $\mathrm{Fe}_{\mathrm{hcp}}$ and the Ho (Fig. 3a). For each pair, we performed ISTS on both atoms (Fig. 3b,c). Remarkably, we observe the following two points: first, the spectra of the coupled Ho atoms do not change regardless of separation or applied magnetic field (Fig. 3 and Supplementary Note 4), and second the spectra of the coupled $\mathrm{Fe}_{\mathrm{hcp}}$ atom also do not change for separations larger than $5.55 \AA$ (Fig. 3). It was previously shown that a signature of exchange fields between a pair of atoms can be detected as a perturbation to the spin excitation $^{20}$. The lack of any significant changes to the $\mathrm{Fe}$ excitations indicate that the indirect exchange field produced by Ho is negligible at our measurement temperature. Only for the two closest pairs $(d=4.24,5.55 \AA)$ we observe a change in the measurement. There is a drop of the excitation intensity, but the excitation energy remains constant. It is important to note that we further checked the $B$-dependent ISTS measurements up to $7 \mathrm{~T}$ on the closest pair in Supplementary Figure 6 in Supplementary Note 4, which show that the evolution of the spin-excitation of the $\mathrm{Fe}$ in this pair is identical to that of an isolated $\mathrm{Fe}$ atom. The intensity drop is therefore probably an effect due to the modified vacuum density of states rather than due to a magnetic interaction. Taking into account the narrow linewidth of the spin-excitation step in the $\mathrm{Fe}_{\mathrm{hcp}}$ spectrum, we can show that the method has a sensitivity of $50 \mu \mathrm{eV}$ (see Supplementary Figure 7 in Supplementary Note 5). We can thus conclude that the interaction between $\mathrm{Fe}$ and $\mathrm{Ho}$ in the $\mathrm{RKKY}$ regime is smaller than this limit.

Electronic and magnetic structure calculations. To support these experimental findings, we performed DFT calculations for isolated atoms and pairs using the Korringa-Kohn-Rostoker Green function method ${ }^{21}$. Both $\mathrm{Fe}_{\mathrm{fcc}}$ and $\mathrm{Fe}_{\mathrm{hcp}}$ were considered, and spin-orbit coupling as well as an local density approximation $(\mathrm{LDA})+\mathrm{U}$ correction for the $4 f$ states of Ho were included. Further methodological details are given in Supplementary Note 6, and Supplementary Table 2 in Supplementary Note 7 additionally summarizes the ground state properties of both adatom types. In Fig. 4a, we show the spin-polarization, $P(E)=100\left(N_{\uparrow}(E)-N_{\downarrow}(E)\right) /\left(N_{\uparrow}(E)+N_{\downarrow}(E)\right)$ with $N_{\uparrow, \downarrow}(E)$ as the number of spin-up or spin-down electrons at the specified energy $E$, in the vacuum at two different heights from an $\mathrm{Fe}_{\mathrm{fcc}}$ or $\mathrm{Ho}_{\mathrm{fcc}}$ atom. For $\mathrm{Fe}$, a substantial spin-polarization of $\approx 10 \%$ is obtained for $d=4.5 \AA$, increasing up to $30 \%$ for $d=2.8 \AA$. This results from the spin-polarized $3 d$ states of $\mathrm{Fe}$ in this energy window. Conversely, the spin-polarization of Ho is below $1 \%$, as the Ho $4 f$ density of states is low and not spin-polarized in this energy window. As it is well-known that magnetic atoms can induce a large moment in surrounding $\mathrm{Pt}$ atoms ${ }^{3,14}$, we calculated the net moment induced in $\mathrm{Pt}$ by $\mathrm{Fe}_{\mathrm{fcc}}$ and $\mathrm{Ho}_{\mathrm{fcc}}$ independently. For $\mathrm{Fe}$, the induced spin moment is $0.72 \mu_{\mathrm{B}}$ (in Khajetoorians et al. ${ }^{14} \mathrm{a}$ larger cluster was used, leading to an induced spin moment of $1.02 \mu_{\mathrm{B}}$ (see Supplementary Note 6 for details)). This points to a strong magnetic coupling between the Fe $3 d$ orbitals and the Pt $5 d$ and $6 s$ states. For Ho, we get an induced moment of $0.05 \mu_{\mathrm{B}}$, reinforcing the weak hybridization picture for the $4 f$ states.

Figure $4 \mathrm{~b}$ illustrates the calculated distance-dependent indirect exchange interaction between three different types of hcp-hcp pairs, namely $\mathrm{Fe}-\mathrm{Fe}, \mathrm{Fe}-\mathrm{Ho}$ and $\mathrm{Ho}-\mathrm{Ho}$. The magnetic coupling of $\mathrm{Fe}-\mathrm{Ho}$ or Ho-Ho pairs is nearly 100 times smaller than that of the corresponding $\mathrm{Fe}-\mathrm{Fe}$ pair at the same distance. These results corroborate the experimental findings in Fig. 3, in which there is no evidence of indirect exchange interactions produced by a Ho atom. The root of the weak exchange field produced by Ho can be illustrated by investigating the electronic structure of the Ho atom in comparison to the Fe atom (Fig. 4c). The minority Fe $3 d$ orbitals are relatively close to the Fermi energy $E_{\mathrm{F}}$, while the Ho $4 f$ orbitals were pushed away by the LDA + U correction. Overall, the $4 f$ states of Ho appear sharper than the $3 d$ states of Fe located at similar energies, suggesting a weaker hybridization for $\mathrm{Ho}-\mathrm{Pt}$ than for Fe-Pt. The magnetic coupling of a dimer is affected by virtual transitions between the $3 d$ or $4 f$ orbitals of the different atoms, which are sensitive to both the position of the peaks with respect to $E_{\mathrm{F}}$ and the strength of the hybridization (see Supplementary Figure 8 in Supplementary Note 6 to find a graph showing the dependency of the coupling strength to the $U$ correction in the LDA $+\mathrm{U}$ calculation). This results in a much weaker Fe-Ho coupling as compared with the $\mathrm{Fe}-\mathrm{Fe}$ coupling, which is below the experimental sensitivity. Likewise, the tunnelling conductance is affected by the same quantities in a similar way (see Supplementary Note 8 ). This implies that the inelastic excitation intensity for Ho is very weak in comparison to $\mathrm{Fe}$, that is, the coupling of tunnelling electrons to the localized $4 f$ levels is less probable as compared with the hybridized $3 d$ levels, suggesting why there is an absence of inelastic signal in ISTS. The $4 f$ hybridization with its electronic environment is overestimated due to insufficient spatial localization of the $4 f$ orbitals. This may be better described by a LDA + DMFT type approach, which may also better describe the full multiplet structure of $\mathrm{Ho} /$ $\operatorname{Pt}(111)^{22,23}$

\section{Discussion}

In summary, our investigations show that the $4 f$ orbitals of a Ho atom adsorbed on $\mathrm{Pt}(111)$ are very well-isolated from the surrounding electronic and magnetic environment. This has profound implications on atomic-scale magnetic technologies based on rare-earth magnets, as conventional wisdom suggests that magnetic order emerges in rare-earth compounds via 
a

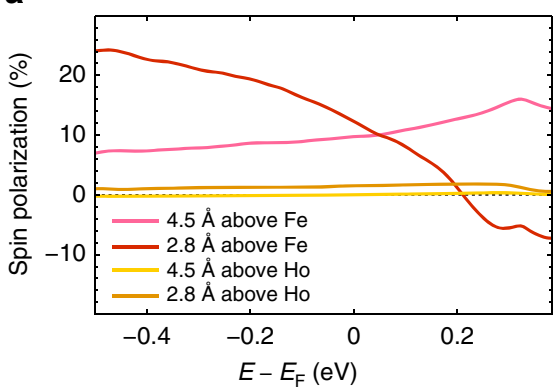

C

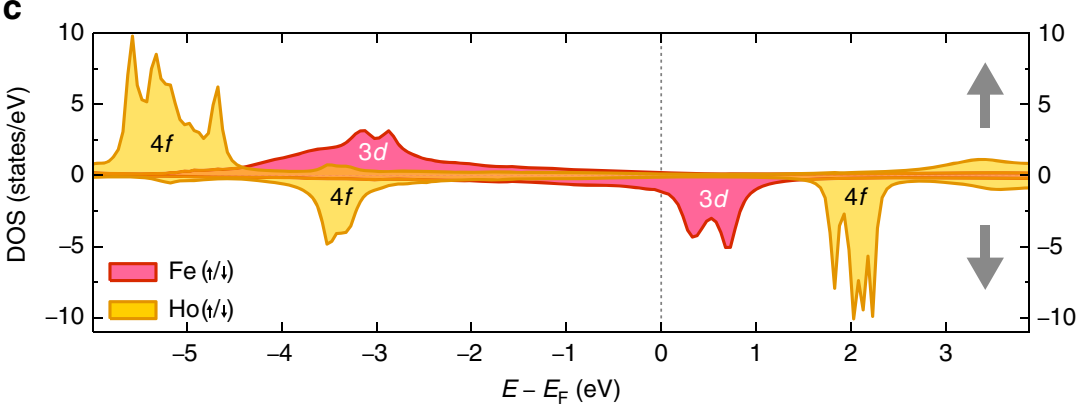

b

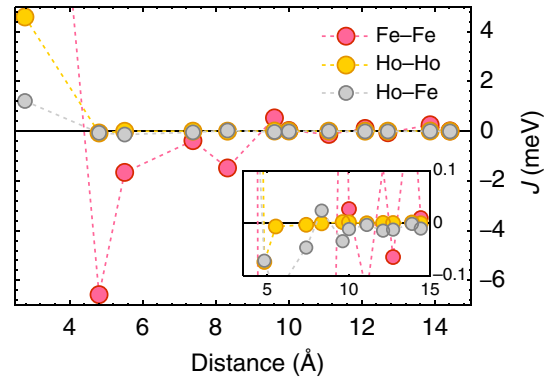

10 0

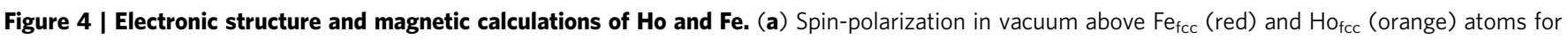

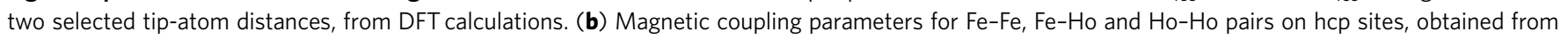

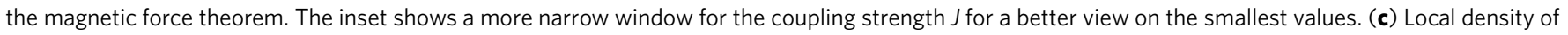

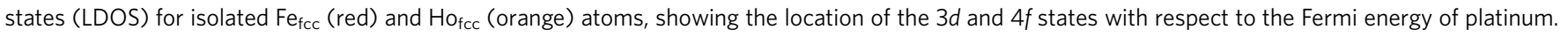
Positive or negative values indicate majority or minority spins, which is also indicated by the grey up or down arrows.

coupling pathways between the $4 f$ orbitals and itinerant electrons. Our results show that these exchange paths are altered if a single rare-earth atom is adsorbed on a surface, most likely due to the change in coordination and symmetry of the atom compared with the bulk. With regards to the work of Miyamachi et al. ${ }^{8}$, the absence of a magnetic and inelastic signal observed here only allows us to speculate about the discrepancy between recent measurements and if the described theoretical proposal was realized, as neither the proper values of the spin-resolved differential conductance, the inelastic intensity nor the sensitivity limits were given in ref. 8. Assuming we have similar detection limits, the observed discrepancy evokes the question, whether the Ho adsorbates investigated here and by Miyamachi et al. ${ }^{8}$ could be of different chemical composition. In addition to clean Ho atoms, we observed adsorbates with a different apparent height which we attribute to hydrogen-contaminated Ho. Moreover, the $\mathrm{Pt}(111)$ surface contains residual defects, which are often located close to Ho atoms. Hydrogen or other contaminations of adatoms can either strongly change their magnetism ${ }^{24}$ or lead to conformational changes ${ }^{25}$ that can be confused with magnetic excitations in ISTS or magnetic switching in spin-polarized measurements. Before our measurements, we therefore always removed hydrogen from the surface and the adatoms and manipulated the adatom to a clean spot of $\mathrm{Pt}(111)$ to exclude such effects. The absence of telegraph noise rules out a ground state of $J_{z}=8$ for the clean Ho atoms we investigate, in contrast to ref. 8 , and corroborates a $J_{z}=6$ ground state as found by XMCD measurements by Donati et al. ${ }^{13}$. However, we do not observe the ISTS signal expected from any of the anisotropy values for the spin-Hamiltonian in refs 8,13 . Our investigation therefore rather questions if tunnelling-based methods can probe $4 f$-derived magnetic atoms, due to the weak interaction and strong localization of the $4 f$ orbitals, and if $\mathrm{Ho} / \mathrm{Pt}(111)$ is the proper system to experimentally investigate the theoretical proposal of Miyamachi et al. ${ }^{8}$.

\section{Methods}

STM/STS measurements. The experiments were performed in a home-built STM facility ${ }^{26}$ at a temperature of $T=0.3 \mathrm{~K}$ in a magnetic field $B$ applied perpendicular to the sample surface. We used flashed $\mathrm{W}$ tips with a nominal coating of 50 monolayers of $\mathrm{Cr}$ for spin-polarized measurements ${ }^{4}$. Magnetic contrast was achieved by picking up a number of $\mathrm{Fe}$ atoms, whereas we used a combination of voltage-pulsing and tip-dipping for inelastic ISTS measurements to get feature-less tips within the measured energy range. Constant-current images were recorded at a tunnelling current $I_{\text {stab }}$ with a bias voltage $V_{\mathrm{s}}$ applied to the sample. ISTS was performed by adding a modulation voltage $V_{\text {mod }}$ (r.m.s.) of frequency $f_{\text {mod }}$ to $V_{\mathrm{s}}$, stabilizing the tip at $I_{\text {stab }}$ and $V_{\text {stab }}$, switching the feedback off, ramping the bias voltage and recording the $\mathrm{d} I / \mathrm{d} V$ signal using a lock-in amplifier with time constant $\tau$ and sensitivity $V_{\text {sens. }}$. The $\operatorname{Pt}(111)$-crystal was cleaned in situ as described in ref. 14. Ho atoms were evaporated onto the surface from a Knudsencell at a temperature of $900^{\circ} \mathrm{C}$ for $20 \mathrm{~s}$ and a base pressure of $9 \times 10^{-11} \mathrm{mbar}$. Afterwards, single Fe atoms were co-deposited onto the same sample using an e-beam evaporator with an $\mathrm{Fe}$ rod. During deposition, the sample temperature did not exceed $20 \mathrm{~K}$. The result is a statistical distribution of Ho and Fe atoms on the $\mathrm{Pt}(111)$ surface (Supplementary Note 1 and Supplementary Figure 1).

Electronic and magnetic structure calculations. We used the Korringa-KohnRostoker Greeen function method ${ }^{21}$ for the DFT calculations of single Fe and Ho atoms both on hcp and fcc lattice sites. Spin-orbit coupling was included and a $\mathrm{LDA}+\mathrm{U}$ correction was applied for the $4 f$ states of Ho. A slab of 22 Pt layers stacked in the (111) direction augmented by two vacuum regions was used to define the undisturbed $\mathrm{Pt}(111)$ surface. Both the Fe and Ho atoms were relaxed vertically towards the surface by $20 \%$. To correctly take into account for the hybridization of $d$ and $f$ states with itinerant surface electrons, the Anderson impurity model in the mean-field approximation was used. For more details about the performed calculations, we would like to refer the reader to the provided Supplementary Notes 6-8.

\section{References}

1. Khajetoorians, A. A., Wiebe, J., Chilian, B. \& Wiesendanger, R. Realizing allspin-based logic operations atom by atom. Science 332, 1062-1064 (2011).

2. Rau, I. G. et al. Reaching the magnetic anisotropy limit of a $3 \mathrm{~d}$ metal atom. Science 344, 988-992 (2014).

3. Gambardella, P. et al. Giant magnetic anisotropy of single cobalt atoms and nanoparticles. Science 300, 1130-1133 (2003).

4. Khajetoorians, A. A. et al. Itinerant nature of atom-magnetization excitation by tunnelling electrons. Phys. Rev. Lett. 106, 037205 (2011). 
5. Khajetoorians, A. A. et al. Current-driven spin dynamics of artificially constructed quantum magnets. Science 339, 55-59 (2013).

6. Loth, S., Baumann, S., Lutz, C. P., Eigler, D. \& Heinrich, A. J. Bistability in atomic-scale antiferromagnets. Science 335, 196-199 (2012).

7. Spinelli, A., Bryant, B., Delgado, F., Fernandez-Rossier, J. \& Otte, A. F. Imaging of spin waves in atomically designed nanomagnets. Nat. Mater. 13, 782-785 (2014).

8. Miyamachi, T. et al. Stabilizing the magnetic moment of single holmium atoms by symmetry. Nature 503, 242-246 (2013).

9. Karlewski, C. et al. Magnetic adatoms as memory bits: a quantum master equation analysis. Phys. Rev. B 91, 245430 (2015).

10. Hübner, C., Baxevanis, B., Khajetoorians, A. A. \& Pfannkuche, D. Symmetry effects on the spin switching of adatoms. Phys. Rev. B 90, 155134 (2014).

11. Jensen, J. \& Mackintosh, A. R. Rare Earth Magnetism: Structures and Excitations (Clarendon Press, 1991).

12. Coffey, D. et al. Antiferromagnetic spin coupling between rare earth adatoms and iron islands probed by spin-polarized tunnelling. Sci. Rep. 5, 13709 (2015).

13. Donati, F. et al. Magnetism of ho and er atoms on close-packed metal surfaces. Phys. Rev. Lett. 113, 237201 (2014).

14. Khajetoorians, A. A. et al. Spin excitations of individual fe atoms on pt(111): Impact of the site-dependent giant substrate polarization. Phys. Rev. Lett. 111, 157204 (2013)

15. Heinrich, A. J., Gupta, J. A., Lutz, C. P. \& Eigler, D. M. Single-atom spin-flip spectroscopy. Science 306, 466-469 (2004).

16. Fernández-Rossier, J. Theory of single-spin inelastic tunnelling spectroscopy. Phys. Rev. Lett. 102, 256802 (2009).

17. Schweflinghaus, B., dos Santos Dias, M., Costa, A. T. \& Lounis, S. Renormalization of electron self-energies via their interaction with spin excitations: A first-principles investigation. Phys. Rev. B 89, 235439 (2014).

18. Meier, F. et al. Spin polarization of platinum (111) induced by the proximity to cobalt nanostripes. Phys. Rev. B 83, 075407 (2011).

19. Zhou, L. et al. Strength and directionality of surface ruderman-kittel-kasuyayosida interaction mapped on the atomic scale. Nat. Phys. 6, 187-191 (2010).

20. Otte, A. F. et al. Spin excitations of a kondo-screened atom coupled to a second magnetic atom. Phys. Rev. Lett. 103, 107203 (2009).

21. Papanikolau, N., Zeller, R. \& Dederichs, P. Conceptual improvements of the kkr method. J. Phys. Condens. Matter 14, 2799-2823 (2002).

22. Lebegue, S., Svane, A., Katsnelson, M. I., Lichtenstein, A. I. \& Eriksson, O. Multiplet effects in the electronic structure of heavy rare-earth metals. J. Phys. Condens. Matter 18, 6329 (2006).

23. Gardonio, S. et al. Excitation spectra of transition-metal atoms on the ag (100) surface controlled by hund's exchange. Phys. Rev. Lett. 110, 186404 (2013).

24. Khajetoorians, A. A. et al. Tuning emergent magnetism in a hund's impurity. Nat. Nanotechnol. 10, 958-964 (2015).
25. Ternes, M., Heinrich, A. J. \& Schneider, W.-D. Spectroscopic manifestations of the kondo effect on single adatoms. J. Phys. Condens. Matter 21, 053001 (2009).

26. Wiebe, J. et al. A $300 \mathrm{mK}$ ultra-high vacuum scanning tunnelling microscope for spin-resolved spectroscopy at high energy resolution. Rev. Sci. Instrum. 75, 4871-4879 (2004).

\section{Acknowledgements}

We would like to thank C. Hübner and H. Brune for helpful discussions. M.S., A.S., J.W. R.W. and A.A.K. acknowledge funding from the SFB668 and the GrK1286 of the DFG M.d.S.D., M.B. and S.L. acknowledge funding from the HGF-YIG Programme FunSiLab (VH-NG-717) and the DFG project LO 1659/5-1. A.S. and A.A.K. also acknowledge funding from the Emmy Noether Programme of the DFG (KH324/1-1). A.A.K. also acknowledges funding from FOM, an organization of NWO, and the NWO-Vidi programme.

\section{Author contributions}

A.A.K., J.W. and M.S. designed the experiment. M.S. and A.S. carried out the measurements. M.d.S.D. and M.B. performed the calculations. M.S., M.d.S.D., A.S., J.W. and A.A.K. wrote the manuscript. M.S. prepared the figures. All authors contributed to the discussion and interpretation of the results, as well as the discussion of the manuscript.

\section{Additional information}

Supplementary Information accompanies this paper at http://www.nature.com/ naturecommunications

Competing financial interests: The authors declare no competing financial interests.

Reprints and permission information is available online at http://npg.nature.com/ reprintsandpermissions/

How to cite this article: Steinbrecher, M. et al. Absence of spin-signature of a single Ho adatom as probed by spin-sensitive tunnelling. Nat. Commun. 7:10454 doi: $10.1038 /$ ncomms10454 (2016).

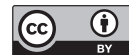

This work is licensed under a Creative Commons Attribution 4.0 International License. The images or other third party material in this article are included in the article's Creative Commons license, unless indicated otherwise in the credit line; if the material is not included under the Creative Commons license, users will need to obtain permission from the license holder to reproduce the material. To view a copy of this license, visit http://creativecommons.org/licenses/by/4.0/ 